\section{ALLELIC VARIATION IN HUMAN LEUKOCYTE ANTIGEN CLASS II GENES IS ASSOCIATED WITH PNEUMONITIS RISK IN CANCER PATIENTS TREATED WITH IMMUNE CHECKPOINT INHIBITORS}

${ }^{1}$ Ashis Saha, ${ }^{2}$ Christine Spencer, 'Zia Khan, ${ }^{1} J o n a t h a n$ Carroll, ${ }^{3}$ Claudia Yanez Arellano, ${ }^{1} J u l i e$ Hunkapiller, ${ }^{2}$ Nicholas Bayless, ${ }^{2}$ Leonardo Nissola, ${ }^{4}$ Roslyn Wallace, ${ }^{1}$ Ira Mellman, ${ }^{3}$ Rajat Mohindra, ${ }^{2}$ Samantha Bucktrout, ${ }^{4}$ Shahneen Sandhu, ${ }^{3} \mathrm{G}$. Scott Chandler, ${ }^{1}$ Christian Hammer*, ${ }^{1}$ Christian Hammer. 'Genentech, South San Francisco, CA, United States; ${ }^{2}$ Parker Institute f. Cancer Immunotherapy, San Francisco, CA, United States; ${ }^{3} \mathrm{~F}$. Hoffmann-La Roche, Basel, Switzerland; ${ }^{4}$ Peter MacCallum Cancer Centre, Melbourne, Australia

Background Immune-mediated adverse events (imAE) commonly occur in patients treated with immune checkpoint inhibitors (ICI), and pneumonitis is known to occur in $3-5$ $\%$ of patients treated with anti-PD-1 / PD-L1 antibodies. ${ }^{1}$ Most cases are grade 1 or 2 events and can be treated with immunosuppression, but high-grade events occur in a minority of patients and can be fatal. ${ }^{2}$ Since lung inflammatory phenotypes, including fibrotic idiopathic interstitial pneumonias and infectious pneumonias, were associated with allelic variation in Human Leukocyte Antigen (HLA) genes, ${ }^{3}{ }^{4}$ we hypothesized that HLA variants might also be a risk factor for ICI- associated pneumonitis.

Methods Out of 1761 atezolizumab (anti-PD-L1) treated patients across nine Genentech (GNE) clinical trials with available whole-genome sequencing data, 72 (4.1\%) developed pneumonitis (table 1). We inferred HLA genotypes using HLA-HD ${ }^{5}$ and performed an association study including 87 alleles with a carrier frequency of $>2 \%$. In order to confirm our results, and to test whether the association is generalizable to different classes of ICI, we genotyped two additional cohorts using an Illumina genome-wide SNP array (GSA v3), followed by HLA imputation using $\mathrm{HIBAG}^{6}$ : (1) 20 ICItreated cancer patients with pneumonitis and 20 matched controls without from a pilot study on the AEROSMITH trial from Parker Institute for Cancer Immunotherapy (PICI); (2) 15 ICI-treated melanoma patients with pneumonitis and 149 without from Peter MacCallum Cancer Centre (PMC) (table 1).

Results Two HLA class II alleles that are part of a common haplotype showed significant associations with pneumonitis risk after multiple testing adjustment (HLA-DRB1*15:01, HLA-DQA1*01:02), with HLA-DRB1*15:01 showing the strongest association $(\mathrm{p}=0.0002$, odds ratio $(\mathrm{OR})=2.51)$. No associations were identified in the control arms $(\mathrm{N}=$ 1192). In the PICI pilot cohort, HLA-DRB1*15:01 did not reach statistical significance in spite of a comparable OR ( $\mathrm{p}=$ 0.26 , OR $=2.75$ ), but the allele was significantly associated with pneumonitis risk in the PMC cohort $(\mathrm{p}=0.03, \mathrm{OR}=$ 3.92). A meta-analysis across the three cohorts yielded a highly significant $\mathrm{p}$-value of $1.2 \times 10-5(\mathrm{OR}=2.67$, figure 1$)$, suggesting that the association is generalizable across ICI. Importantly, the same class II haplotype was previously shown to be associated with diverse lung inflammatory, including fibrotic, phenotypes. ${ }^{3,4,7,8}$

Abstract 811 Table 1 Investigated cohorts
\begin{tabular}{|l|l|l|l|l|l|l|}
\hline Cohort & $\begin{array}{l}\text { ICl-associated } \\
\text { pneumonitis }\end{array}$ & \multicolumn{5}{l}{ Treatment } \\
\hline & yes & no & PD-L1 & PD-1 & CTLA4 & PD-1 + CTLA-4 \\
\hline GNE & 72 & 1689 & 1761 & 0 & 0 & 0 \\
\hline PICl & 19 & 19 & 14 & 22 & 0 & 2 \\
\hline PMC & 15 & 149 & 0 & 47 & 14 & 103 \\
\hline Total & 106 & 1857 & 1775 & 69 & 14 & 105 \\
\hline
\end{tabular}

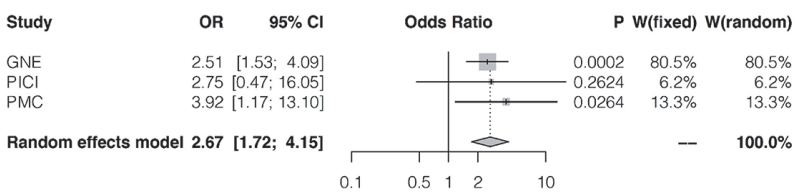

Abstract 811 Figure 1 Forest plot for HLA-DRB1*15:01 meta-analysis with ICl-associated pneumonitis. GNE, Genentech; $\mathrm{PICl}$, Parker Institute for Cancer Immunotherapy; PMC, Peter MacCallum Cancer Centre; OR, odds ratio; $\mathrm{Cl}$, confidence interval; W, weight http://dx.doi.org/10.1136/ jitc-2021-SITC2021.811

Conclusions In summary, our findings establish HLA class II allelic variation as a potential risk factor in ICI-associated pneumonitis, and future research is warranted to determine whether this genetic association can be refined according to specific clinical presentations.

\section{REFERENCES}

1. Wang H, Guo X, Zhou J, Li Y, Duan L, Si X, et al. Clinical diagnosis and treatment of immune checkpoint inhibitor-associated pneumonitis. Thorac Cancer 2020:11:191-7.

2. Naidoo J, Wang X, Woo KM, Iyriboz T, Halpenny D, Cunningham J, et al. Pneumonitis in patients treated with anti-programmed death-1/Programmed death ligand 1 therapy. J Clin Oncol 2016:35:709-17.

3. Tian C, Hromatka BS, Kiefer AK, Eriksson N, Noble SM, Tung JY, et al. Genomewide association and HLA region fine-mapping studies identify susceptibility loci for multiple common infections. Nat Commun 2017;8:599.

4. Fingerlin $\mathrm{TE}$, Zhang $\mathrm{W}$, Yang IV, Ainsworth $\mathrm{HC}$, Russell $\mathrm{PH}$, Blumhagen RZ, et al. Genome-wide imputation study identifies novel HLA locus for pulmonary fibrosis and potential role for auto-immunity in fibrotic idiopathic interstitial pneumonia. BMC Genet 2016:17:74

5. Kawaguchi S, Higasa K, Shimizu M, Yamada R, Matsuda F. HLA-HD: An accurate HLA typing algorithm for next-generation sequencing data. Hum Mutat 2017;38:788-97.

6. Zheng X, Shen J, Cox C, Wakefield JC, Ehm MG, Nelson MR, et al. HIBAG-HLA genotype imputation with attribute bagging. Pharmacogenomics J 2014;14:192200.

7. Voorter CEM, Drent M, Berg-Loonen EM van den. Severe pulmonary sarcoidosis is strongly associated with the haplotype HLA-DQB1*0602-DRB1*150101. Hum Immunol 2005:66:826-35.

8. Furukawa H, Oka S, Shimada K, Sugii S, Ohashi J, Matsui T, et al. Association of human leukocyte antigen with interstitial lung disease in rheumatoid arthritis: a protective role for shared epitope. Plos One 2012;7:e33133.

Ethics Approval Patients included in this study signed an optional Research Biosample Repository (RBR) Informed Consent Form (ICF) and provided whole blood samples. By signing the optional RBR ICF, patients provided informed consent for analysis of inherited and non-inherited genetic variation from whole blood samples. Ethics Committees (EC) and Institutional Review Boards (IRB) in each country and each study site for each clinical trial approved the clinical trial protocol, the main study ICF, and theRBR ICF.

http://dx.doi.org/10.1136/jitc-2021-SITC2021.811 